\title{
Small Intestinal Cancer Diagnosed by Video Capsule Endoscopy and Double-Balloon Enteroscopy
}

\author{
Shutaro Ozawa ${ }^{1)}$, Masami Yamada ${ }^{1)}$, Mineo Hanawa ${ }^{1)}$ and Isamu Koyama ${ }^{2)}$ \\ ${ }^{1)}$ Department of Surgery, Chichibu Hospital \\ ${ }^{2)}$ Department of Digestive Surgery, Saitama Medical University International Medical Center
}

\begin{abstract}
A 41-year-old man presented with general fatigue, tarry stool and epigastralgia. Hematological examination data showed an anemia ( $\mathrm{Hb} 9.3 \mathrm{~g} / \mathrm{dl})$. No abnormalities were found on upper gastrointestinal endoscopy and colonoscopy. Therefore patient was received a video capsule endoscopy. A stenosis with reddish and edematous mucosal changes was recognized at the upper jejunum. Furthermore, doubleballoon enteroscopy was added to this patient. Pathological studies of the biopsy specimens diagnosed a primary small intestinal adenocarcinoma. Computed tomography showed the regional lymph node metastasis without distant metastasis. The patient was received a radical operation. Primary small intestinal adenocarcinoma is a rare malignancy ; most cases cannot be detected on routine gastrointestinal endoscopy due to their location. Therefore, it is often that diagnosis is late. Our experience suggests that double-balloon enteroscopy and video capsule endoscopy are useful for diagnosing small intestinal adenocarcinoma.
\end{abstract}

Key words: double-balloon enteroscopy, video capsule endoscopy, primary small intestinal carcinoma

\section{Introduction}

Small bowel cancer is relatively rare malignancy as compared with other malignancies of the gastrointestinal tract. In fact, small bowel cancer only ac-

Received: October 3, 2011/Accepted: October 16, 2011

Correspondence to: Shutaro Ozawa

Department of Surgery, Chichibu Hospital, 20 Izumicho, Chichibu-shi, Saitama 369-1874, Japan count for $1-5 \%$ of all gastrointestinal tract tumors ${ }^{11}$. The reason of this rarity of these tumors, combined with the presence of non-specific signs and symptoms, can delay prompt diagnosis and treatment $t^{1,2)}$. The early detection of lesions in the small intestine has been hindered by their inaccessibility to endoscopy and their limitations of radiographic techniques ${ }^{1,2)}$.

Video capsule endoscopy (VCE), which enables the whole small bowel observation safely and no invasively, was introduced in $2000^{3)}$. Double-balloon enteroscopy (DBE) has been developed in Japan ${ }^{4)}$. DBE allows high-resolution visualization, as well as diagnostic and therapeutic interventions, for all segments of small intestine ${ }^{5}$. In this case, DBE and VCE were useful for preoperative diagnosis of small intestinal adenocarcioma.

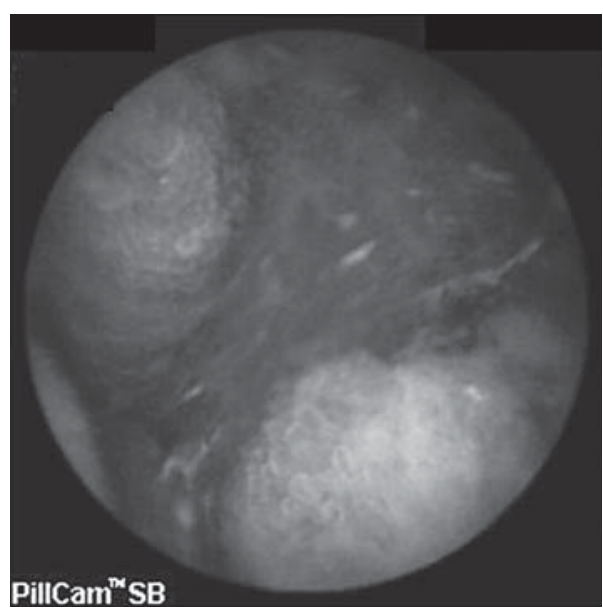

Fig. 1 VCE showed that a stenosis with reddish and edematous mucosal changes was recognized at the upper jejunum. 


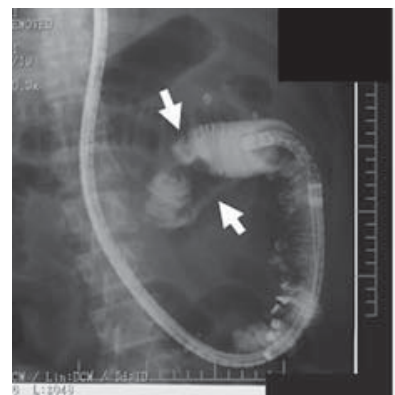

A

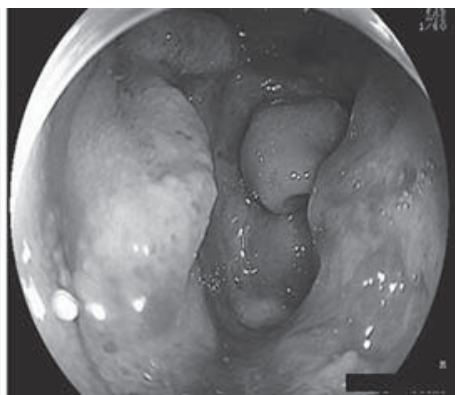

B

Fig. 2 DBE was performed to confirm the $x$-ray findings.

A : X-ray showed that stenosis of jejunum was recognized in the $30 \mathrm{~cm}$ anus side from a Treitz' ligament (allows showed the point of stenosis).

B : DBE revealed a type2 cancer at the same point.

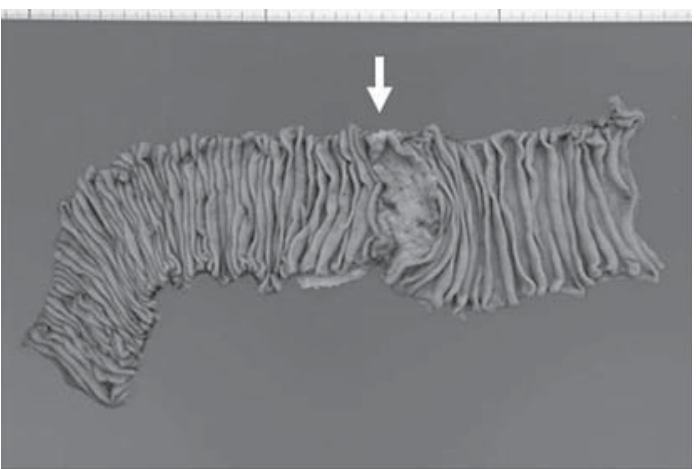

Fig. 3 Upper jejunum was removed partially. Allow showed the tumor with stenosis and diameter was $5.5 \times 2.5 \mathrm{~cm}$. The tumor invaded into the layer of subserosa and surgical margin was cancer negative. Two regional lymph node metastases were recognized.

\section{Case Report}

A 41-year-old man was receiving a treatment for hepatitis $\mathrm{C}$ in private hospital. He presented with 3-month history of general fatigue and 1-month history of tarry stool and epigastralgia. No abnormalities were found on upper gastrointestinal endoscopy and colonoscopy. He was introduced to our hospital for diagnosis. There were no abnormalities in physical examination. Admission hematological data showed an anemia ( $\mathrm{Hb} 9.3 \mathrm{~g} / \mathrm{dl}$ ). CEA was $1.1 \mathrm{ng} / \mathrm{mL}$ and CA19-9 was $2.6 \mathrm{U} / \mathrm{ml}$ (within normal). Therefore, he received a VCE. A stenosis with reddish and edematous mucosal changes was recognized at the

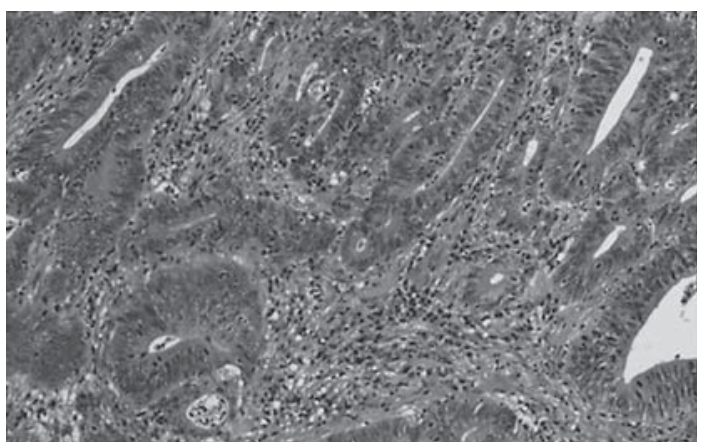

Fig. 4 The pathological finding was well differentiated adenocaricinoma $(H \& E \times 200)$.

upper jejunum (Fig. 1 ). Furthermore, DBE was performed to confirm the x-ray findings and to obtain a tissue diagnosis. DBE showed that tumor was present in the $30 \mathrm{~cm}$ anus side from a Treitz' ligament (Fig.2A, B).

On histological examination of the biopsy specimens, well differentiated adenocarcinoma was noted. Computed tomography showed the regional lymph node metastasis without distant metastasis. Based on these findings, he was diagnosed as advanced primary small intestinal cancer. Given that patient presented with stage III disease, we proposed a radical operation. Operative findings showed that there was neither peritoneal dissemination nor hepatic metastasis. Cancer was present in the $30 \mathrm{~cm}$ anus side from a Treitz' ligament and the tumor was egg of hen size. The second jejunal artery supply blood flow to tumor. Therefore, we exposed super mesen- 
teric artery and legated the second jejunal artery in a root for a sufficient lymphatic dissection. We performed the partial resection of jejunum while taking distance enough from cancer (Fig. 3 ). We anastomosed residual jejunum in instrument and finished operation without drain. The pathological finding was well differentiated adenocaricinoma (Fig. 4) which invaded into the layer of subserosa. The diameter of cancer was $5.5 \times 2.5 \mathrm{~cm}$ and the form of tumor was type2. There were a few regional lymph node metastases. The postoperative course was uneventful, and he was discharged on postoperative day 7 .

\section{Discussion}

Adenocarcinoma of small intestine is a rare tumor and to our knowledge few published studies in the literature to date have addressed it adequately. In one of the largest series published to date from the SEER program duodenal tumors were shown to be more common (48\%) than tumors of the jejunum (23 $\%)$ and ileum (16\%) and that $30 \%$ of patients present with metastatic disease ${ }^{5)}$.

The small bowel is generally considered as a rare location for the development of neoplasms, accounting for only $1-5 \%$ of all primary gastrointestinal tumors ${ }^{1,6)}$. Primary adenocarcinoma is the most common histological subtype of carcinoma of the small intestine, constituting $40 \%$ of cases. Carcinoid tumors are the second most common type, accounting for $36 \%$ of cases ${ }^{5)}$.

The most common symptom of small bowel cancer is pain, followed by gastrointestinal bleeding, weight loss, nausea, and vomiting ${ }^{1,2}$. The current patient had some of these symptoms. The nonspecific nature of symptoms usually leads to delay in presentation and diagnostic workup ${ }^{5)}$. Adenocarcinoma of the small intestine carries a dismal prognosis. Lioe et al. reported that over half of patients had lymphatic metastases and distant metastases at that time of diagnosis. 5-year survival was around $15 \%{ }^{1)}$. The median age of the affected patients is 50-60 years old. Moreover, the younger patients tend to have more advanced disease than the older patients ${ }^{7}$. The current case was relative young and had an advanced stage with some lymphatic metastases without distant metastasis.

Moreover, due to their location, most lesions cannot be detected by routine gastrointestinal endosco- pies $^{2)}$. Thus, investigation of the small intestine has been ultimate challenge for gastroenterologists. VCE was introduced and it enables the whole small bowel observation safely and no invasively ${ }^{3)}$. Recently, DBE has been developed in Japan ${ }^{4}$. Compared to conventional investigation, such as push endoscopy and small bowel barium radiography, VCE has been reported to have a higher yield ${ }^{8)}$. However, VCE is not without its limitations. Chong et al. described 4 patients in whom small bowel pathology was missed on VCE but was detected on $\mathrm{DBE}^{9)}$. These studies suggested that DBE may have a greater yield than VCE. However VCE is a noninvasive tool, well accepted by patients, who can allow the visualization of the entire small bowel ; high-quality images of the small-bowel mucosa may be captured and small and flat lesions recognized, without exposure to radiation ${ }^{10)}$. Nevertheless, DBE examinations are more labor intensive, require the use of sedation, and may not allow the small intestine to be completely examined from either the antegrade or the retrograde approach alone. There are demerits and merits in VCE and DBE.

In this case, we use VCE as a surveillance and $\mathrm{DBE}$ as a diagnosis for preoperative analysis. Thus, VCE and DBE may be considered to be complementary.

\section{References}

1) Lioe TF, Biggart JD : Primary adenocarcinoma of the jejunum and ileum ; clinicopathological review of 25 cases. J Clin Pathol 43 : 533-536, 1996

2) Cunningham JD, Aleali R, Aleali $\mathrm{M}:$ Malignant small bowel neoplasms. Ann Surg 225 : 300-306, 1997

3) Iddan G, Meron G, Glukhovsky A, et al : Wireless capsule endoscopy. Nature $25: 405: 417,2000$

4) Yamamoto H, Sekine $Y$, Sato $Y$, et al : Total eneteroscopy with nonsurgical steerable double-ballon method. Gastrointest Endosc 53 : 216-220, 2001

5) Chow JS, Chen CC, Ahsan H, et al : A populationbased study of the incidence of malignant small bowel tumors : SEER, 1973-1990. Int J Epidemiol 25 : 722-728, 1996

6) Neugut AI, Jacobson JS, Suh S, et al : The epidemiology of cancer of the small bowel. Cancer Epidemiol Biomarkers Prev $7: 243-251,1998$

7) Dabaja BS, Suki D, Pro B, et al : Adenocarcinoma of the small bowel : presentation, prognostic factors, and outcome of 217 patients. Cancer $101: 518-526$, 2004

8) Triester SL, Leighton JA, Leontiadis GI, et al : A meta-analysis of the yield of capsule endoscopy compared to other diagnostic modalities in patients with non-stricturing small bowel Crohn's disease. Am J 
Adenocaricnoma of small intestine

Gastroenterol 101 : 954-964, 2006

9) Chong AK, Chin BW, Meredith CG : Clinically significant small-bowel pathology identified by double-balloon enteroscopy but missed by capsule endoscopy.
Gastrointest Endosc 64 : 445-449, 2006

10) Pennazio M, Rondonotti E, de Franchis R : Capsule endoscopy in neoplastic diseases. World J Gastroenterol $14: 5245-5253,2008$ 